\title{
Transitions from Near-Surface to Interior Redox upon Lithiation in Conversion Electrode Materials
}

Kai He ${ }^{\dagger, \mathbb{A}}$, Huolin L. Xin ${ }^{\dagger, \pi}$, Kejie Zhao ${ }^{\ddagger}$, Xiqian $\mathrm{Yu}^{\S}$, Dennis Nordlund", Tsu-Chien Weng", Jing $\mathrm{Li}^{\dagger}{ }^{\dagger}$, , Yi Jiang ${ }^{\#}$, Christopher A. Cadigan ${ }^{\nabla}$, Ryan M. Richards ${ }^{\nabla}$, Marca M. Doeff ${ }^{\circ}$,Xiao-Qing Yang $^{\S}$, Eric A. Stach ${ }^{\dagger}, \mathrm{Ju} \mathrm{Li}^{\star, *}$, Feng Lin ${ }^{\nabla, \circ, *}$, and Dong Su ${ }^{\dagger, \perp, *}$

${ }^{\dagger}$ Center for Functional Nanomaterials, Brookhaven National Laboratory, Upton, New York 11973, USA.

Department of Materials Science and Engineering, Massachusetts Institute of Technology, Cambridge, Massachusetts 02139, USA.

${ }^{\S}$ Chemistry Department, Brookhaven National Laboratory, Upton, New York 11973, USA.

"Stanford Synchrotron Radiation Lightsource, SLAC National Accelerator Laboratory, Menlo Park, California 94025, USA.

${ }^{\perp}$ Department of Materials Science and Engineering, Stony Brook University, Stony Brook, New York 11794, USA.

"Department of Physics, Cornell University, Ithaca, New York 14853, USA.

${ }^{\nabla}$ Department of Chemistry and Geochemistry, Materials Science Program, Colorado School of Mines, Golden, Colorado 80401, USA.

${ }^{\circ}$ Environmental Energy Technologies Division, Lawrence Berkeley National Laboratory, Berkeley, California 94720, USA.

${ }^{\mathbb{I}} \mathrm{K} . H$. and H.L.X. contributed equally to this work.

*E-mails: liju@mit.edu; flin@lbl.gov; dsu@bnl.gov

ABSTRACT: Nanoparticle electrodes in lithium-ion batteries have both near-surface and interior contributions to their redox capacity, each with distinct rate capabilities. Using combined electron microscopy, synchrotron X-ray methods and ab initio calculations, we have investigated the lithiation pathways that occur in $\mathrm{NiO}$ electrodes. We find that the near-surface electroactive $\left(\mathrm{Ni}^{2+} \rightarrow \mathrm{Ni}^{0}\right)$ sites saturated very quickly, and then encounter unexpected difficulty in propagating the phase transition into the electrode (referred to as a "shrinking-core" mode). However, the interior capacity for $\mathrm{Ni}^{2+} \rightarrow \mathrm{Ni}^{0}$ can be accessed efficiently following the nucleation of lithiation "fingers" which propagate into the sample bulk, but only after a certain incubation time. Our microstructural observations of the transition from a slow shrinking-core mode to a faster lithiation finger mode corroborate with synchrotron characterization of large-format batteries, and can be rationalized by stress effects on transport at high-rate discharge. The finite incubation time of the lithiation fingers sets the intrinsic limitation for the rate capability (and thus the power) of $\mathrm{NiO}$ for electrochemical energy storage devices. The present work unravels the link 
between the nanoscale reaction pathways and the C-rate-dependent capacity loss, and provides guidance for the further design of battery materials that favors high C-rate charging.

KEYWORDS: lithium ion battery, nickel oxide, conversion reaction, in situ TEM, incubation, rate capability

Lithium-ion batteries and supercapacitors both rely on electrochemical redox processes, although different mechanisms determine their relative energy and power densities ${ }^{1-5}$. For nanostructured electrodes of lithium-ion batteries, the capacity contains contributions from redox reactions that occur in both the interior (I) and near-surface (NS) regions ${ }^{6-9}$. It is believed that the interior redox reactions contribute more to the overall battery capacity, but these take a longer time to be activated. In contrast, redox reactions in the near-surface reaction may exhibit a supercapacitor-like behavior (i.e. a high power density) because of the short transport paths for ions and electrons ${ }^{7-9}$. Thus, an understanding of the kinetics of the transition from NS-redox to Iredox is critical to determining the rate capability of a lithium ion battery. Even though the rate capability and reaction kinetics can be improved by modifications to the material structure (such as surface coating and functionalization) and by architecture engineering ${ }^{10-21}$, it is still not clear how the redox front propagates into the interior, especially at high discharge rates.

In this work, we have integrated complementary atomic resolution transmission electron microscopy (TEM) imaging, electron and X-ray spectroscopies, and electrochemistry measurements to study the kinetics of the lithiation of $\mathrm{NiO}$ nanosheet electrodes. After visualizing the heterogeneous redox reaction modes at the near-surface and interior regions, we found that the transition from NS-redox to I-redox reaction is exceptionally slow when it occurs through the classic "shrinking-core" mode ${ }^{22}$. Instead, most of the interior $\mathrm{Ni}^{2+} \rightarrow \mathrm{Ni}^{0}$ sites are mined only after high-aspect-ratio "reaction fingers" are nucleated. These subsequently penetrate deeply into the nanoparticle interior and then thicken so as to spread the transition through the bulk of the material. There is an "incubation" time, which averages to about 100s from in situ TEM observations, before the redox reaction can penetrate into the interior. The reaction timescale and patterns we discovered from in situ TEM correlate with the ultimate rate performance of large-format batteries and are further supported by ex situ TEM and X-ray spectroscopies. We believe such heterogeneous transition mechanisms from NS-redox to I-redox may be generic and transferrable to a large class of conversion nano-electrode materials.

Our experiments were performed using three setups: (I) 2032-type coin cells (Figure 1a,b) using liquid electrolyte, (II) TEM “grid-in-a-coin-cell” using liquid electrolyte, for easy postmortem TEM characterization of large-format batteries (Figure 1c-g), and (III) in situ lithiation 
(Figure 2, 3) using $\mathrm{Li}_{2} \mathrm{O}$ solid electrolyte. Setups I, II, III all use the same NiO nanosheets with holes and faceted surfaces ${ }^{23,24}$ as positive electrodes, and Li metal as negative electrodes. For battery performance the initial formatting cycle is crucial, as the structures inherited from the first cycle may dramatically influence subsequent cycles for conversion electrodes ${ }^{25}$. Therefore, the first cycle, specifically the first lithiation process, as shown in reaction Equation $(1)^{26,27}$ and confirmed by in situ diffraction (Supporting Information Figure S1 and Movie 4), is the focus of the present paper.

$$
\mathrm{NiO}+2 \mathrm{Li}^{+}+2 \mathrm{e}^{-}(U) \rightarrow \mathrm{Ni}+\mathrm{Li}_{2} \mathrm{O}
$$

For setup I, the voltage-total capacity curves are shown in Figure 1a for high ( 10C) and low $(\sim 0.1 \mathrm{C})$ charging rate, respectively. The nominal first-cycle discharge (lithiation) capacities obtained at 10C $(800 \mathrm{mAh} / \mathrm{g})$ and $0.1 \mathrm{C}(1160 \mathrm{mAh} / \mathrm{g})$ are both larger than the theorical specific capacity $(718 \mathrm{mAh} / \mathrm{g})$ of $\mathrm{NiO}$. This does not mean full conversion of $\mathrm{Ni}^{2+} \rightarrow \mathrm{Ni}^{0}$ centers ${ }^{28}$, however, due to electrolyte decomposition and SEI formation which consumes injected electrons: these side reactions become possible for ethylene carbonate/diethyl carbonate liquid electrolyte whenever the voltage $U$ drops below $\sim 1.3 \mathrm{~V}$ against $\mathrm{Li}^{+} / \mathrm{Li}$ (ref. 3). To reveal the actual degree of $\mathrm{Ni}^{2+} \rightarrow \mathrm{Ni}^{0}$ conversion in the first discharge, we performed synchrotron hard X-ray absorption spectroscopy ${ }^{29,30}$ (XAS, Supporting Information Figure S2) which characterizes the valence state of all Ni atoms contained in a 2032 coin cell. The XAS spectra were fit to a linear combination of $\mathrm{Ni}^{2+}$ and $\mathrm{Ni}^{0}$ spectroscopic components, and the degree of $\mathrm{Ni}^{2+} \rightarrow \mathrm{Ni}^{0}$ conversion is indicated in Figure $1 \mathrm{~b}$ against the Fraction of Total Discharge (FoTD). (Please note that FoTD includes side reactions). It is interesting to note that at $\mathrm{FoTD}=100 \%$, all the $\mathrm{Ni}^{2+}$ were reduced to $\mathrm{Ni}^{0}$ in the $0.1 \mathrm{C}$ coin cell, but in the $10 \mathrm{C}$ coin cell $\sim 15 \%$ of the $\mathrm{Ni}^{2+}$ still remained unreduced. This means that at $10 \mathrm{C}$ not all the electroactive sites in $\mathrm{NiO}$ nanosheets can be reduced even as the voltage dropped to near zero against $\mathrm{Li}^{+} / \mathrm{Li}$ (the equilibrium voltage of Equation (1) is $0.6 \mathrm{~V}$ against $\left.\mathrm{Li}^{+} / \mathrm{Li}\right){ }^{25}$ This measures the true rate-dependent electrodic capacity of NiO.

It is also interesting to note that very little $\mathrm{Ni}^{2+}$ were reduced up to FoTD $=25 \%$, and $0.1 \mathrm{C}$ and 10C samples do not differ much in this regard (Figure 1b). This means electrolyte decomposition and SEI formation consumes most of electrons injected up to the first quarter of discharge, which is reasonable since SEI formation happens below $U=1.3 \mathrm{~V}^{3}$, earlier than $U_{0}=$ $0.6 \mathrm{~V}^{25}$ for reaction (1). For $10 \mathrm{C}$ sample, even rather limited amount of $\mathrm{Ni}^{2+} / \mathrm{Ni}^{0}$ redox happened at the NS region (as observed by TEM), which is difficult to be detected due to the low (surface) sensitivity of hard X-ray and the quantification technique using spectral linear combination. But SEI formation kinetics is self-slowing, after the SEI thickness has grown. Generally speaking, SEI formation dominates the early part of the first discharge, and 0.1C sample forms more SEI than the 10C sample $(1160-718=442 \mathrm{mAh} / \mathrm{g}$ vs. $800-718 \times 0.85=190 \mathrm{mAh} / \mathrm{g}$, so almost double by the end of the discharge) overall due to the longer reaction time. For the later part of the first discharge, $\mathrm{Ni}^{2+} \rightarrow \mathrm{Ni}^{0}$ conversion dominates the total reaction. The difference between $0.1 \mathrm{C}$ and 
10C samples in this regard is that while the degree of $\mathrm{Ni}^{2+} \rightarrow \mathrm{Ni}^{0}$ is almost linear between FoTD $=$ $25 \%$ to $100 \%$ at $0.1 \mathrm{C}$, for the $10 \mathrm{C}$ sample the $\mathrm{Ni}^{2+} \rightarrow \mathrm{Ni}^{0}$ reduction kinetics seems to have an nonlinear behavior. In the $10 \mathrm{C}$ sample, there is a small amount of conversion from FoTD $=25 \%$ to $50 \%$, followed by very rapid conversion between FoTD $=50 \%$ to $75 \%$ ("inflection"). As $10 \mathrm{C}$ means 6 minutes at full discharge: this "inflection" stage is quite remarkable in the sense that more than $50 \%$ of all the $\mathrm{Ni}^{2+}$ in the coin cell is reduced in a time duration $\tau_{\text {infection }} \sim 1.5$ minutes. This indicates that certain intrinsic kinetics in $\mathrm{NiO}$ nanosheets ${ }^{23,24}$ must be quite fast to be able to accomplish this, at nearly a constant voltage $\sim 0.4 \mathrm{~V}$ (this driving voltage $U$ barely changed between FoTD $=0$ to $75 \%$ ). Thus, the "intrinsic" rate capability of $\mathrm{NiO}$ nanosheets ${ }^{23,24}$ can be quite $\operatorname{good}^{6}$. But for the FoTD $=75 \%$ to $100 \%$, for various reasons (e.g. electrolyte decomposition, long-range $\mathrm{Li}^{+} /$electron transport disruptions, or another possible reason to be suggested later) the previously rapid "inflection" slowed down, even though the driving voltage $U$ drops more rapidly in the last quarter of discharge, and thus increases the reductive driving force. The $\mathrm{Ni}^{2+} \rightarrow \mathrm{Ni}^{0}$ conversion ended up unfinished, leaving behind $15 \%$ unreduced $\mathrm{Ni}^{2+}$. Such nonlinear "incubation-infection-saturation" coin-cell behavior at timescale of minutes may thus be attributed to an intrinsic $\mathrm{NiO}$ electrodic timescale, as opposed to the $0.1 \mathrm{C}$ case where the electron supply is throttled externally to be much slower than $\tau_{\text {infection. }}$.

We note that a redox timescale of minutes at $10 \mathrm{C}$ is on the borderline between what is nominally considered supercapacitor-like behavior and battery behavior $\left(\tau_{\text {infection }} \sim 1.5\right.$ minute would be considered fast in the battery context, but slow in the redox supercapacitor context). Conventional understanding of the operation of lithium ion batteries involves consideration of both near-surface electroactive sites and interior electroactive sites. However, there are few direct characterizations of either the NS or I behaviors and observations of the NS $\rightarrow$ I transition are few. In order to understand how discharge rate actually drives changes in the material's microstructure, we have utilized the TEM "grid-in-a-coin cell" method ${ }^{23}$ as illustrated in Figure 1c (setup II). This approach allows us to subject $\mathrm{NiO}$ to states of charge that mimic the situation in a fully operational battery, yet are directly amenable to TEM imaging. The pristine NiO materials are $\sim 20$-nm-thick sheets, with closely-packed $\{111\}$ basal planes forming the largest free surfaces, and they are initially perforated with hexagonal holes that are bounded by <112> facets enabling short diffusion paths from NS to I redox sites (Figure 1d and Supporting Information Figure S3 ${ }^{23,24}$. The uniform and thin thickness is natively electron-transparent and thus suitable for in situ TEM observations, particularly for large-scale in-plane lithiation propagation. The discharged samples usually contained a significant fraction of unreacted $\mathrm{NiO}$ (unreduced $\mathrm{Ni}^{2+}$ ), with the reacted regions consisting of fine metallic $\mathrm{Ni}$ nanoparticles (Supporting Information Figure $\mathrm{S} 4$ ) embedded in $\mathrm{Li}_{2} \mathrm{O}$ matrix or covering the $\mathrm{NiO}$ surface (Figure 1e-g). Critically, for both C-rates we found three distinct, heterogeneous morphologies, but which differed in their relative amounts. We call these three lithiation modes as Modes bulk (B), shrinking-core (S), and finger-like (F), and describe their appearance in detail below. 
In Mode B (bulk mode), the reaction front has advanced deeply inside the $\mathrm{NiO}$ interior (Figure 1e) with the reaction front normal $\boldsymbol{n} \perp$ [111]. The reaction front is relatively flat, exhibits no strong crystallographic preference in its propagation direction (except for $\perp$ [111]), and is sharply bounded by unreacted $\mathrm{NiO}$. In Mode S (shrinking-core mode), the reaction appears to have first proceeded very rapidly along all surfaces by surface diffusion (later verified by in situ observations), and then thickens further uniformly perpendicular to all surfaces - including the (111) surfaces ${ }^{22}$ (Figure 1f). Mode F (finger mode) exhibits a strikingly different morphology: a high-aspect-ratio lithiation finger $\perp$ [111], penetrates deep inside the unreacted material. Mode $F$ has an overall appearance similar to that of a crack or wedge, although its microscopic manifestation is the reaction of $\mathrm{NiO}$ to $\mathrm{Ni}+\mathrm{Li}_{2} \mathrm{O}$, and not the cleavage of a material as would be observed in a crack (Figure $1 \mathrm{~g}$ ). In order to understand the correlation between the reaction morphology and the discharge rates, we imaged a large number of nanosheets reacted ex situ to a specified FoTD $(20 \%, 40 \%, 60 \%, 80 \%$ and $100 \%$, corresponding to the points labeled 'A', 'B', 'C', 'D', 'E' in Figure 1a, respectively), at both $10 \mathrm{C}$ and $0.1 \mathrm{C}$. Figure $1 \mathrm{~h}$ summarizes the statistics obtained from the TEM images, which were collected in an unbiased manner (see an example in Supporting Information Figure S5). We see a distinct difference in the prevalence of different reaction morphologies as a function of the discharge rate: the high C-rate samples show a higher prevalence of Mode S (shrinking-core mode), while the low C-rate favors Mode B (bulk mode). Moreover, as the particle-ensemble FoTD advances, the 10C reaction morphology switched from being primarily Mode S to Mode B.

While a rigorous statistical link is established between the rate and the reaction modes at particles-ensemble level in Setup II, ex post facto imaging of this type cannot provide timedependent trajectory of the NS $\rightarrow$ I reaction transition. In order to investigate this phenomenon further, we have utilized a real-time electron microscopy approach (Setup III) to investigate the reaction kinetics for the different modes. The in situ experiments utilized a dry electrochemical cell, with no side reaction of organic electrolyte decomposition ${ }^{31-33}$. As illustrated in Figure 2a, $\mathrm{Li}$ ions are transferred towards the $\mathrm{NiO}$ electrode under a positive bias in a potentiostatic mode. In the in situ discharging experiments (Supporting Information Movies 1-2), even though there is no deliberate control of the discharge rates, we repeatedly and reproducibly observed the same reaction modes as in the ex situ cells (Figure 2b,c): this indicates that this in situ method is able to provide valid information that can be correlated with the ex post facto observations. In Figure $2 \mathrm{~b}$ as well as Supporting Information Movie 1, we observed that the surface wetting is very fast by surface diffusion and the redox front propagates from the outer surface (after NS sites have been saturated). Such further propagation (via the shrinking core mode), encounters unexpected difficulty when trying to access the interior, and proceeds rather slowly perpendicular to all surfaces of the nanosheet, including (111) surfaces. 
However, we also found other pathways for the NS $\rightarrow$ I transition through the nucleation and subsequent growth of lithiation fingers, which occurred heterogeneously throughout the sheet (Figure 2c). The fingers initially nucleate on <112> facets randomly, a while after the nearsurface sites saturate, then elongate $\perp$ [111] until the exits on the opposite surface of the $\mathrm{NiO}$ nanosheet. After penetrating all the way through, then thicken into the nanosheet bulk. At the fully discharged state, the fingers have thickened enough to completely merge and therefore few identifiable fingers can be seen at FoTD $=100 \%$. From the in situ observations, we found that Mode B occurred after the fingers in Mode $\mathrm{F}$ have thickened significantly and/or merged. We thus propose that Mode B occurs after the fingers have formed and thus facilitated $\mathrm{Li}^{+} / \mathrm{e}^{-}$supply through the interior. After the formation of fingers, the long-range transport capability through interior of the $\mathrm{NiO}$ nanosheets is improved and thus promotes the transition to Mode $\mathrm{B}$. At no time were the nanosheets broken. This is in contrast to observations of true material fracture observed in Si nanoparticle $\mathrm{e}^{34,35}$ and $\mathrm{ZnO}$ nanowire ${ }^{36}$ anode materials.

The complete picture of the reaction pathways for $\mathrm{NiO}$ nanosheets is depicted in Figure $2 \mathrm{~d}$, where two types of transitions from NS $\rightarrow$ I are observed: the shrinking-core mode and the finger mode, whereas the $\mathrm{I} \rightarrow \mathrm{I}$ transition is proceeded through bulk mode (Mode B, Supporting Information Movie 3). The fact that more Mode B events are observed in low-rate discharged sample implies that, after more finger penetrations have been established, the lithiation process is promoted. Using Supporting Information Movies 1-2, a quantitative analysis of the front propagation speed reveals a large difference between the shrinking-core and the finger modes, as shown in Figure 2e and Supporting Information Figure S6. The finger mode, after the finger has nucleated, propagates at average speed $0.045 \mathrm{~nm} / \mathrm{s}$, which is an order of magnitude faster than the shrinking-core mode. Therefore, we can deduce that the NS $\rightarrow \mathrm{I}$ transition is more likely to happen via the finger mode than the shrinking-core mode, after the surfaces have reached a point where the near-surface sites are fully saturated. There appears to be an energy barrier against $\mathrm{NS} \rightarrow$ I transition, which is lower for the more heterogeneous finger mode than for the shrinkingcore mode. Before the finger nucleates, there appears to be an incubation time: we saw that the fingers generally do not form immediately after saturation of the near surface sites. We have collected the statistics of finger nucleation, shown in Figure $2 \mathrm{f}$, and found that the characteristic incubation time $\tau_{\text {incubation }}$ - defined as the average time lapse for the first finger to appear after near-surface site saturation in an area containing 1 hole on average (taken to be the characteristic in-plane length scale of the $\mathrm{NiO}$ nanosheet, around 30nm) - is 100 seconds. During the incubation, Li ions were transported to an interior redox site at near-surface and accumulated until nucleating a lithiation finger. This single-particle timescale matches reasonably well with the aforementioned coin-cell intrinsic timescale $\tau_{\text {incubation }} \sim 1.5$ minute $($ FoTD $=0$ to $25 \%$ ) for Setup I. Therefore, a possible interpretation of the XAS results in Figure1b is that in our coincell particle ensemble, after the surface/interfacial transport channels are activated and saturated, 
it takes time for the fingers to nucleate, elongate and thicken to access the interior capacity. At $0.1 \mathrm{C}$ (36,000 seconds for a full discharge), there is plenty of time for fingers to nucleate, so the microstructural evolution is throttled externally by electron supply. But at 10C (360 seconds discharge), a significant fraction of the characteristic in-plane areas will not be able to incubate

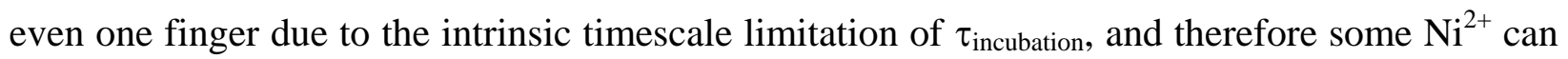
remain unreduced at FoTD $=100 \%$ even as the voltage is drastically lowered. With this assumption, the natural demarcation on the time axis to separate supercapacitor-like behavior from battery-like behavior would be $\tau_{\text {discharge }} \sim 100$ s, or around $30 \mathrm{C}$. This means that the interior capacity can only be substantially utilized for galvanostatic rates slower than 30C; otherwise only the near-surface capacity can be utilized. A verifiable prediction from our theory is therefore that if the galvanostatic rate is higher than $30 \mathrm{C}$, our $\mathrm{NiO}$ nanosheets can only be used in the "supercapacitor" fashion, meaning that they exhibit a much reduced capacity (viewed in the battery context), even if the long-range transport limitation associated with Li ion in organic electrolyte were to be removed ${ }^{37}$. The intrinsic kinetic timescale extracted from our in situ TEM, $\tau_{\text {incubation}}$, does seem to match both our own coin-cell data as well as the general understanding of how $\mathrm{NiO}$ should perform at high rates ${ }^{37}$.

In order to understand the finger nucleation and propagation processes in more detail, we utilized analytical scanning transmission electron microscopy (STEM) to study the Mode F regions, immediately following their formation. Reconstructed three-dimensional (3D) STEM electron tomograms clearly show that the Mode F reaction fronts form deep 3Dpenetrations into the NiO slab (Fig 3a and Supporting Information Movie 5). High magnification STEM images (Figure 3b, e, h) indicate that fingers nucleate and grow into the sample bulk only after the local surfaces have fully saturated. We further explored the reaction chemistry at the nanoscale through electron energy-loss spectroscopic (EELS) mapping (Figure 3c, f, i). These maps indicate that both metallic $\mathrm{Ni}^{0}$ and $\mathrm{Li}^{+}$are present at the sample surface and within the penetration grooves (finger regions). In addition, we have captured an early-phase finger groove at atomic resolution as shown in Figure 3d.

From both ex situ and in situ TEM experiments, we observed that the lithiation of $\mathrm{NiO}$ disfavors propagation along $\langle 111\rangle$. To interpret this, using ab initio calculations based on density functional theory, we calculated the formation energies and the migration energies along the $\langle 100\rangle$, $\langle 110\rangle$, and $\langle 111\rangle$ directions at various Li concentrations (Figure 4a, b). We found that $\mathrm{Li}^{+}$transport along $<100>$ and $<110>$ directions is preferred. From the structural view shown in Figure $4 \mathrm{a}$, it is thus plausible that $\mathrm{Li}$ ions transport more readily along in-plane orientations $(<100\rangle$ and $<110>$ ) where open channels are available for Li-ion insertion, whereas the diffusion pathway along the $\langle 111>$ orientation is blocked by front neighbor atoms.

Given that full lithiation can generate a volumetric strain up to $120 \%$ (Supporting Information Figure S7), the finger mode may be interpreted as a spontaneous symmetry-breaking 
instability (Figure 4c) in which the effect of self-stress on Li-ion diffusion plays a key role in perpetuating the long-aspect ratio growth, until the lithiation finger reaches the opposite surface, at which point it has to thicken. In contrast, the slow kinetics of the shrinking-core mode can be understood from a finite deformation model (Supporting Information Figure S8 and S9). In the circumferential (or core-shell) mode, the material is under three-dimensional constraints, which induces a triaxial stress field. This regulates the reaction in a more significant manner. This is consistent with recent studies that showed that a sufficiently large pressure at the interface of crystalline silicon could stop the lithiation reaction ${ }^{38}$. Stress, which is closely related to grain boundaries and other structural defects, may also affect short-range electron transfer ${ }^{39}$ and phase boundary mobility ${ }^{40}$, and stress-relief induced contact network disruptions can alter the longrange transport routes of electrons and ions. Thus the overall evolution can be quite complicated in an actual battery where conductive carbon black, binder and the size and morphology of active material particles and the presence of the SEI all could play significant roles. Nonetheless, we believe the intrinsic electrodic timescale limitation measured by both our in situ, and postmortem method, $\tau_{\text {incubation }} \sim 100$ seconds and $\tau_{\text {infection }} \sim 1.5$ minute, has an important bearing on both "high-rate" batteries as well as potentially on "low-rate" redox supercapacitors. We also identified that local stresses can be used to improve reaction kinetics, which can be leveraged as a new design rule for electrode materials engineering for high C-rate lithium ion battery systems. Our findings of surface-to-bulk phenomenon and rate-dependent lithiation mechanisms in $\mathrm{NiO}$ system could be generically applicable to conversion electrode materials, though optimization of morphologies, facets and sizes may be further helpful to improve redox kinetics. 


\section{Methods}

\section{Sample preparation.}

The active material ( $\mathrm{NiO}$ nanosheets) was synthesized using a solvothermal method aided with an alcohol pseudo-supercritical drying technique ${ }^{24} \cdot \mathrm{Ni}\left(\mathrm{NO}_{3}\right)_{2} \cdot 6 \mathrm{H}_{2} \mathrm{O}$, urea and benzyl alcohol were added into $50 \mathrm{~mL}$ of methanol with a molar ratio of 2:1:4. The solution was stirred for 1 hour and transferred to an autoclave (Parr Reactor, model \#4520). The reaction mixture was purged with Ar for 1 min and then Ar was filled with a pressure of 9 bars before initiating the heating process. The mixture was heated to $265^{\circ} \mathrm{C}$ and maintained for 1.5 hours. Finally, the vapor inside was vented (i.e., pseudo-supercritical drying process). A green powder was collected and subsequently calcined at $500{ }^{\circ} \mathrm{C}$ for 6 hours to yield $\mathrm{NiO}$ nanosheets.

\section{Electrochemical measurements.}

Composite electrodes were prepared with $80 \mathrm{wt} \%$ active material, $10 \mathrm{wt} \%$ polyvinylidene fluoride (PVDF) and 10wt\% acetylene carbon black in N-methyl-2-pyrrolidone (NMP) and cast onto copper current collectors. 2032-type coin cells were assembled in an argon-filled glove box using the composite electrode as the positive electrode and $\mathrm{Li}$ metal as the negative electrode. A Celgard separator 2400 and $1 \mathrm{M} \mathrm{LiPF}_{6}$ electrolyte solution in 1:1 w/w ethylene carbonate/diethyl carbonate were used to fabricate coin cells. Battery testing was performed on a computer controlled VMP3 channels (BioLogic). 1C was defined as full discharging $\mathrm{NiO}$ in 1 hour, which corresponds to a theoretical specific current density of $718 \mathrm{~mA} / \mathrm{g}$.

\section{Synchrotron XAS.}

Ni K-edge XAS measurements were carried out at beamline X18A (NSLS, BNL) in transmission mode using a Si (111) double-crystal monochromator detuned to the 35\% value of its original maximum intensity to eliminate the high order harmonics ${ }^{28}$. X-ray absorption near edge structure (XANES) data was analyzed by ATHENA software package. The linear combination fitting (LCF) by using the spectra of two end members ( $\mathrm{NiO}$ and $\mathrm{Ni}$ ) as the standards achieved the accurate $\mathrm{Li}$ stoichiometry in $\mathrm{Li}_{\mathrm{x}} \mathrm{NiO}_{2}$ at each reaction stage, illustrated by Figure $1 \mathrm{~b}$.

\section{TEM characterization.}

The in situ TEM electrochemical cell was incorporated into a Nanofactory TEM-STM specimen holder (Figure 2a), in which $\mathrm{NiO}$ nanosheets dispersed onto a TEM half-grid with amorphous carbon support are analogous to the NiO-C composite electrode, Li metal is coated onto a piezodriven $\mathrm{W}$ probe as the counter electrode, with a thin layer of $\mathrm{Li}_{2} \mathrm{O}$ formed on $\mathrm{Li}$ metal as the solid electrolyte. The $\mathrm{Li}$ and $\mathrm{NiO}$ were loaded onto the holder in an Ar-filled glovebox and then transferred to TEM column using a sealed Ar bag to avoid air exposure. During the in situ electrochemical tests, a constant negative DC potential was applied to $\mathrm{NiO}$ electrode against the $\mathrm{Li}$ source during the lithiation process, and the lithiation processes were captured by real-time imaging in either TEM or STEM mode. Electron tomography of the in situ lithiated sample was acquired in ADF-STEM mode with tilt series from $-70^{\circ}$ to $70^{\circ}$ and $1^{\circ}$ intervals. The ex situ samples after discharge in coin cells were examined at a large number of lithiated locations to reveal statistical probability of surface or bulk reaction modalities. The total area we checked for each sample was about a few hundreds of micrometer by a few hundreds of micrometer. The in situ measurements and tomography were performed on a JEOL $2100 \mathrm{~F}$ TEM operated at $200 \mathrm{kV}$. 
The high-resolution imaging and analytical EELS were conducted on a Hitachi HD2700C STEM operated at $200 \mathrm{kV}$ and equipped with a probe aberration corrector (spatial resolution $<1 \AA$, energy resolution $0.35 \mathrm{eV}$ ). The 3D tomography was reconstructed using the e-Tomo software suite written by Robert Hovden et al. (Cornell Muller group) and visualized by Avizo 6.3.

\section{Theoretical calculations.}

The first-principles calculations based on density functional theory were performed using Vienna ab initio simulation package (VASP). The projector-augmented wave pseudopotentials were employed and the general gradient approximation for the exchange-correlation functional was used. We construct the crystalline $\mathrm{NiO}$ models of [100]-[010]-[001] and [110]-[111]-[112] orientations, which contain 96 atoms with periodic boundary conditions. The atomic structures and system energy are calculated with an energy cutoff of $400 \mathrm{eV}$. In energy optimization calculations, the atomic coordinates, the super-cell shape and volume were relaxed. The energy optimization was considered complete when the magnitude of the force on each atom was smaller than $0.04 \mathrm{eV}^{-1}$.

Finite element analysis and simulations based on continuum theory of finite deformation were performed both analytically and numerically using COMSOL Multiphysics software suits, details see Supporting Information.

\section{ASSOCIATED CONTENT}

\section{Supporting Information}

Additional details of S/TEM, XAS, DFT calculation, and in situ movies. This material is available free of charge via the Internet at http://pubs.acs.org.

\section{AUTHOR INFORMATION}

\section{Corresponding Authors}

*E-mail: liju@mit.edu

* E-mail: flin@lbl.gov

*E-mail: dsu@bnl.gov

\section{Author Contributions}

K.H. and H.L.X. contributed equally to this work. K.H., H.L.X., F.L. and D.S. conceived and designed the experiments. K.H. and D.S. performed the in situ and ex situ S/TEM experiments. H.L.X. performed electron tomography and EELS acquisition and analysis. F.L., X. Y. and X.Q.Y. performed the electrochemical measurements. K.Z. and Ju L. performed the theoretical modeling. F.L., X.Y., X.-Q.Y., D.N. and T.-C.W. performed the synchrotron X-ray measurements. C.A.C., F.L. and R.M.R. synthesized the $\mathrm{NiO}$ samples. Jing L. participated in 
data analysis. Y.J. participated in tomography reconstruction. K.H., H.L.X., Ju L., F.L. and D.S. discussed the scope of the manuscript. K.H. prepared the figures. K. H., D.S., Ju L., H.L.X., F.L., and E.A.S. wrote the manuscript with help from M.M.D. K.H. and H.L.X. contributed equally to this project. D.S. supervised the project. All authors participated in discussion of the results.

\section{Notes}

The authors declare no competing financial interests.

\section{ACKNOWLEGMENTS}

The electron microscopy work (S/TEM, EELS and tomography) was carried out at the Center for Functional Nanomaterials, Brookhaven National Laboratory, which is supported by the U.S. Department of Energy (DOE), Office of Basic Energy Sciences, under Contract No. DESC0012704. The authors acknowledge the technical support from Dr. Steven N. Ehrlich at Beamline X18A of National Synchrotron Light Source at Brookhaven National Laboratory. X.Y. and X.-Q.Y. were supported by the U.S. DOE, the Assistant Secretary for Energy Efficiency and Renewable Energy, Office of Vehicle Technologies under Contract Number DE-AC0298CH10886. The synchrotron X-ray work was partially carried out at the Stanford Synchrotron Radiation Lightsource, SLAC National Accelerator Laboratory, supported by the U.S. DOE under Contract No. DE-AC02-76SF00515. Y.J. is supported by DOE Grant DE-FG0211ER16210. Ju L. acknowledges support by NSF DMR-1240933 and DMR-1120901. Computational time on the Extreme Science and Engineering Discovery Environment (XSEDE) under the grant number TG-DMR130038 is gratefully acknowledged.

\section{REFERENCES}

1. Tarascon, J. M.; Armand, M. Nature 2001, 414, 359-367.

2. Whittingham, M. S. Chem. Rev. 2004, 104, 4271-4301.

3. Goodenough, J. B.; Kim, Y. Chem. Mater. 2010, 22, 587-603.

4. Arico, A. S.; Bruce, P.; Scrosati, B.; Tarascon, J. M.; Van Schalkwijk, W. Nat. Mater. 2005, 4, 366-377.

5. Simon, P.; Gogotsi, Y.; Dunn, B. Science 2014, 343, 1210-1211.

6. Kang, B.; Ceder, G. Nature 2009, 458, 190-193.

7. Conway, B. E. J. Electrochem. Soc. 1991, 138, 1539-1548.

8. Simon, P.; Gogotsi, Y. Nat. Mater. 2008, 7, 845-854.

9. Wang, G.; Zhang, L.; Zhang, J. Chem. Soc. Rev. 2012, 41, 797-828. 
10. Kang, K.; Meng, Y. S.; Breger, J.; Grey, C. P.; Ceder, G. Science 2006, 311, 977-980.

11. Doeff, M. M.; Hu, Y.; McLarnon, F.; Kostecki, R. Electrochem. Soild-State Lett. 2003, 6, A207-A209.

12. Liu, H.; Strobridge, F. C.; Borkiewicz, O. J.; Wiaderek, K. M.; Chapman, K. W.; Chupas, P. J.; Grey, C. P. Science 2014, 344, 1252817.

13. Xu, B.; Fell, C. R.; Chi, M.; Meng, Y. S. Energy Environ. Sci. 2011, 4, 2223-2233.

14. Fong, R.; von Sacken, U.; Dahn, J. R. J. Electrochem. Soc. 1990, 137, 2009-2013.

15. Poizot, P.; Laruelle, S.; Grugeon, S.; Dupont, L.; Tarascon, J. M. Nature 2000, 407, 496-499.

16. Hu, Y. S.; Guo, Y. G.; Sigle, W.; Hore, S.; Balaya, P.; Maier, J. Nat. Mater. 2006, 5, 713-717.

17. Bruce, P. G.; Scrosati, B.; Tarascon. J. M. Angew. Chem. Int. Ed. 2008, 47, 2930-2946.

18. Lee, K. T.; Cho, J. Nano Today 2011, 6, 28-41.

19. Chan, C. K.; Peng, H.; Liu, G.; McIlwrath, K.; Zhang, X. F.; Huggins, R. A.; Cui, Y. Nat. Nanotech. 2008, 3, 31-35.

20. Liu, N.; Lu, Z.; Zhao, J.; McDowell, M. T.; Lee, H. W.; Zhao, W.; Cui. Y. Nat. Nanotech. 2014, 9, 187-192.

21. Zheng, G.; Lee, S. W.; Liang, Z.; Lee, H. W.; Yan, K.; Yao, H.; Wang, H.; Li, W.; Chu, S.; Cui, Y. Nat. Nanotech. 2014, 9, 618-623.

22. Srinivasan, V.; Newman, J. J. Electrochem. Soc. 2004, 151, A1517-A1529.

23. Lin, F.; Nordlund, D.; Weng, T. C.; Zhu, Y.; Ban, C.; Richards, R. M.; Xin, H. L. Nat. Commun. 2014, 5, 3358.

24. Hu, J.; Zhu, K.; Chen, L.; Yang, H.; Li, Z.; Suchopar, A.; Richards, R. Adv. Mater. 2008, 20, 267-271.

25. Cabana, J.; Monconduit, L.; Larcher, D.; Palacin, M. R. Adv. Mater. 2010, 22, E170-E192.

26. Varghese, B. Reddy, M. V.; Zhu, Y.; Lit, C. S.; Hoong, T. C.; Rao, G. V. S.; Chowdari, B. V. R.; Wee, A. Y. S.; Lim, C. T.; Sow, C. H. Chem. Mater. 2008, 20, 3360-3367.

27. Zhou, G.; Wang, D. W.; Yin, L. C.; Li, N.; Li, F.; Cheng, H. M. ACS Nano 2012, 6, 3214-3223. 
28. Hu, Y. Y.; Liu, Z.; Nam, K. W.; Borkiewicz, O. J.; Cheng, J.; Hua, X.; Dunstan, M. T.; Yu, X.; Wiaderek, K. M.; Du, L. S.; Chapman, K. W.; Chupas, P. J.; Yang, X. Q.; Grey, C. P. Nat. Mater. 2013, 12, 1130-1136.

29. Yu, X.; Lyu, Y.; Gu, L.; Wu, H.; Bak, S. M.; Zhou, Y.; Amine, K.; Ehrlich, S. N.; Li, H.; Nam, K. W.; Yang, X. Q. Adv. Energy Mater. 2014, 4, 1300950.

30. Chueh, W. C.; Gabaly, F. E.; Sugar, J. D.; Bartelt, N. C.; McDaniel, A. H.; Fenton, K. R.; Zavadil, K. R.; Tyliszczak, T.; Lai, W.; McCarty, K. F. Nano Lett. 2013, 13, 866-872.

31. He, K.; Zhou, Y.; Gao, P.; Wang, L.; Pereira, N.; Amatucci, G. G.; Nam, K. W.; Yang, X. Q.; Zhu, Y.; Wang, F.; Su, D. ACS Nano 2014, 8, 7251-7259.

32. Liu, X. H.; Wang, J. W.; Huang, S.; Fan, F.; Huang, X.; Liu, Y.; Krylyuk, S.; Yoo, J.; Dayeh, S. A.; Davydov, A. V.; Mao, S. X.; Picraux, S. T.; Zhang, S.; Li, J.; Zhu, T.; Huang, J. Y. Nat. Nanotech. 2012, 7, 749-756.

33. Gu, M.; Kushima, A.; Shao, Y.; Zhang, J. G.; Liu, J.; Browning, N. D.; Li, J.; Wang, C. Nano Lett. 2013, 13, 5203-5211.

34. McDowell, M. T.; Ryu, I.; Lee, S. W.; Wang, C.; Nix, W. D.; Cui, Y. Adv. Mater. 2012, 24, 6034-6041.

35. Liu, X. H.; Liu, Y.; Kushima, A.; Zhang, S.; Zhu, T.; Li, J.; Huang, J. Y. Adv. Energy Mater. 2012, 2, 722-741.

36. Kushima, A.; Liu, X. H.; Zhu, G.; Wang, Z. L.; Huang, J. Y.; Li, J. Nano Lett. 2011, 11, 4535-4541.

37. Feng, L.; Zhu, Y.; Ding, H.; Ni, C. J. Power Sources 2014, 267, 430-444.

38. Zhao, K.; Pharr, M.; Wan, Q.; Wang, W. L.; Kaxiras, E.; Vlassak, J. J.; Suo, Z. J. Electrochem. Soc. 2012, 159, A238-A243.

39. Bai, P.; Bazant, M. Z. Nat. Commun. 2014, 5, 3585.

40. Yang, H.; Huang, S.; Huang, X.; Fan, F.; Liang, W.; Liu, X. H.; Chen, Q. L.; Huang, J. Y.; Li, J.; Zhu, T.; Zhang, S. Nano Lett. 2012, 12, 1953-1958. 

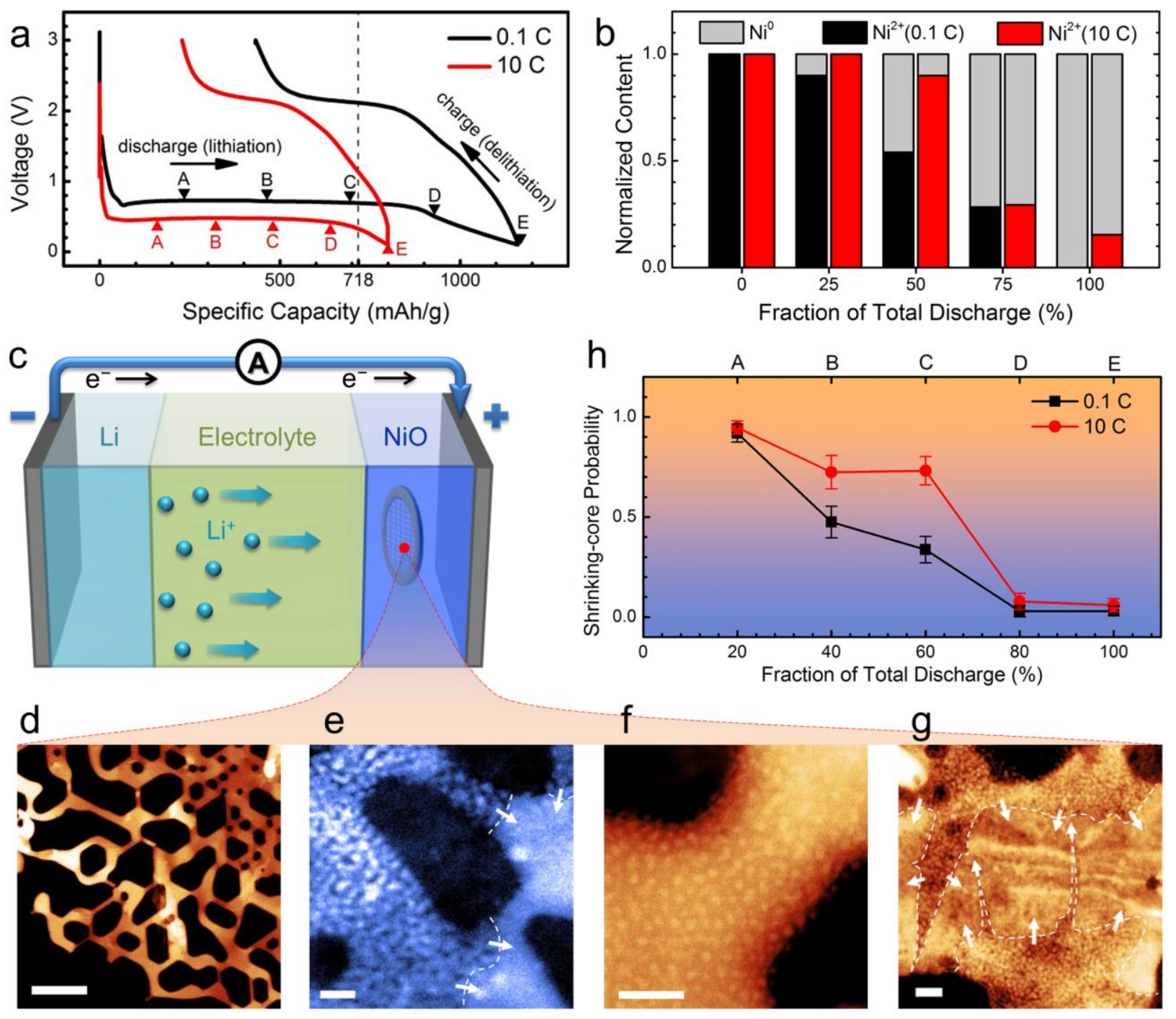

Figure 1. Electrochemical and structural change after lithiation of NiO nanosheets. (a) Chargedischarge curves at rates of $0.1 \mathrm{C}$ and 10C. Dashed line indicates the theoretical capacity of 718 $\mathrm{mAh} / \mathrm{g}$. (b) Relative content of $\mathrm{Ni}^{2+}$ and $\mathrm{Ni}^{0}$ at different discharge fractions as measured by synchrotron Ni K-edge XAS (original spectra shown in Supporting Information Figure2). (c) Schematic illustration of an ex situ battery cell with a TEM grid submerged in the active $\mathrm{NiO}$ electrode. ADF-STEM images of (d) a pristine NiO nanosheet, (e) an area that has almost been fully lithiated via the bulk mode (Mode B), (f) an area that has been partially lithiated only on surface via the shrinking-core mode (Mode $\mathrm{S}$ ), and (g) an area that has been partially lithiated via the finger mode (Mode F). Dashed lines and arrows indicate reaction fronts and propagation directions. (h) Probability of surface mode as a function of discharge fraction via $0.1 \mathrm{C}$ and $10 \mathrm{C}$ discharges. The data points are acquired at the corresponding states labeled A-E in (a). Scale bars, $100 \mathrm{~nm}(\mathrm{~d}), 10 \mathrm{~nm}(\mathrm{e}, \mathrm{f})$, and $20 \mathrm{~nm}(\mathrm{~g})$. 

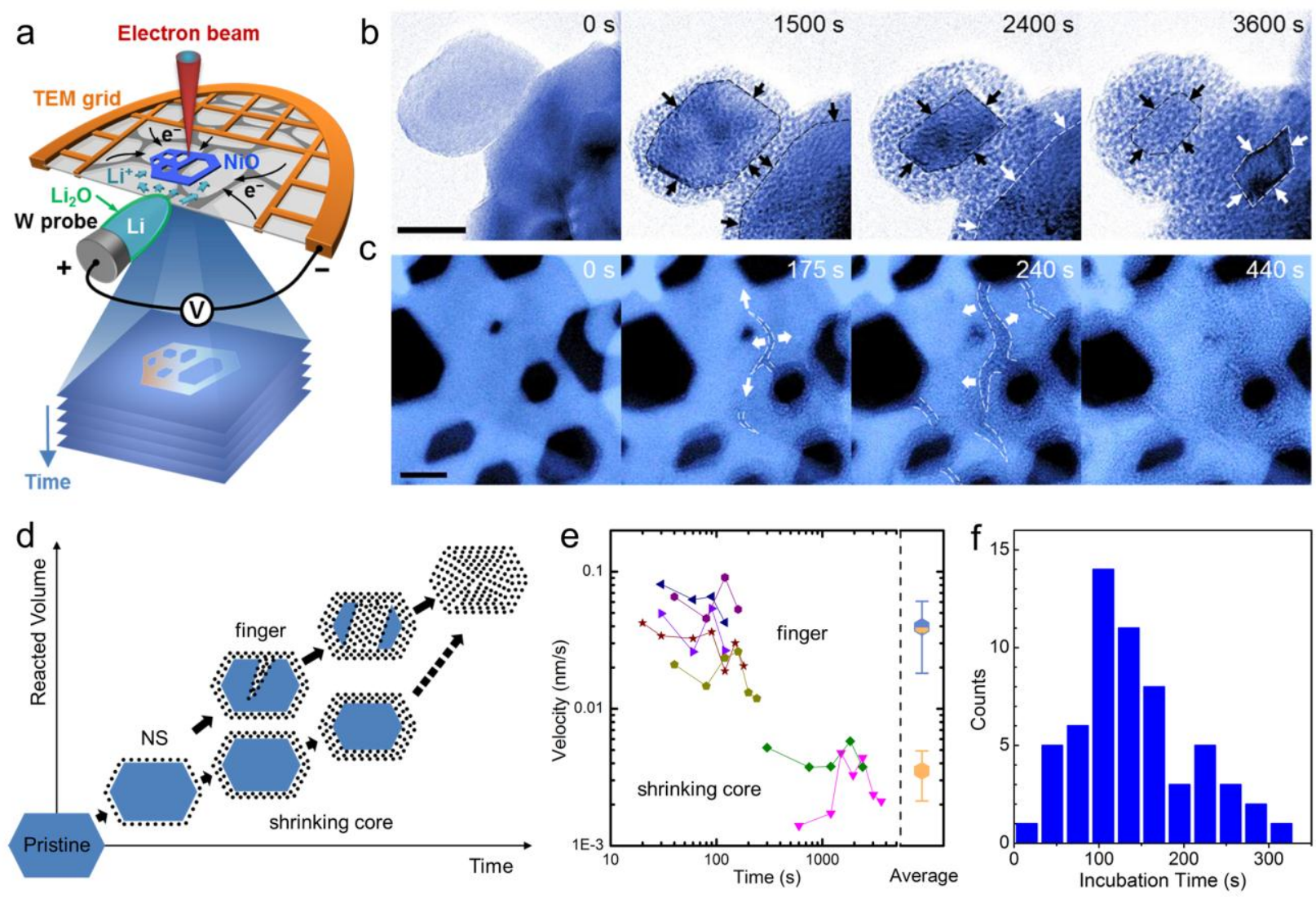

Figure 2. Structural evolution during in situ lithiation of NiO nanosheets. (a) Schematic illustration of in situ electrochemical cell setup. Time-sequenced S/TEM snapshots during in situ lithiation indicate (b) shrinking-core mode, and (c) finger mode. Dashed lines and arrows indicate reaction fronts and propagation directions. (d) Schematic cartoon showing heterogeneous pathways of $\mathrm{N} \rightarrow \mathrm{I}$ transitions. (e) The velocity of reaction front propagation in different pathways deduced from numerous time-dependent position measurements (see Supporting Information Figure 6). The modality-correlated velocities exhibit in two groups and their average values are shown in the right panel. (f) Histogram showing statistics of incubation time from numerous finger-mode events. Scale bars, $20 \mathrm{~nm}$. 

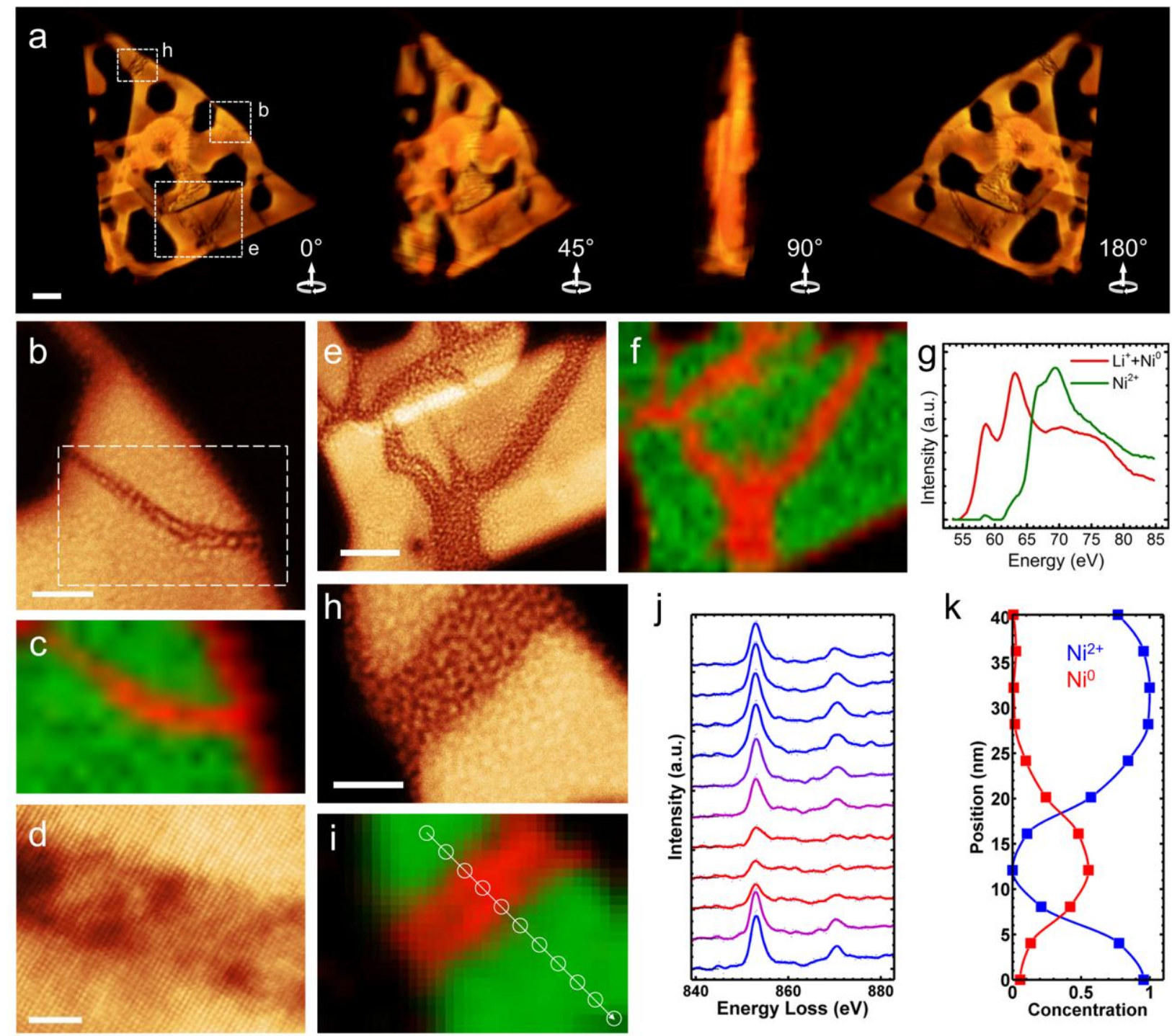

Figure 3. Tomography and EELS mapping of lithiated NiO fingers. (a) A series of reconstructed 3D tomograms from an in situ lithiated $\mathrm{NiO}$ nanosheet at viewing angles of $0^{\circ}, 45^{\circ}$, $90^{\circ}$ and $180^{\circ}$, (also see Supporting Information Movie 5). (b), (e), and (h) are zoom-in ADFSTEM images corresponding to the labeled regions in (a). (c) and (f) show EELS mapping of $\mathrm{Ni}^{2+}$ (green) and $\mathrm{Li}^{+}+\mathrm{Ni}^{0}$ (red) for areas in (b) and (e) using the low-loss spectra components shown in (g). (d) Atomically-resolved STEM image showing a crack region coherently bounded with unlithiated $\mathrm{NiO}$ in (b). (i) EELS charge mapping of $\mathrm{Ni}^{2+}$ (green) and $\mathrm{Ni}^{0}$ (red) for the area in (h), a series of EELS spectra and $\mathrm{Ni}^{2+/ 0}$ concentration profiles along the arrow are shown in (j) and (k), respectively. Scale bars, $20 \mathrm{~nm}$ (a), $10 \mathrm{~nm}$ (b), $2 \mathrm{~nm}$ (d), $20 \mathrm{~nm}$ (e), $10 \mathrm{~nm}$ (h). 


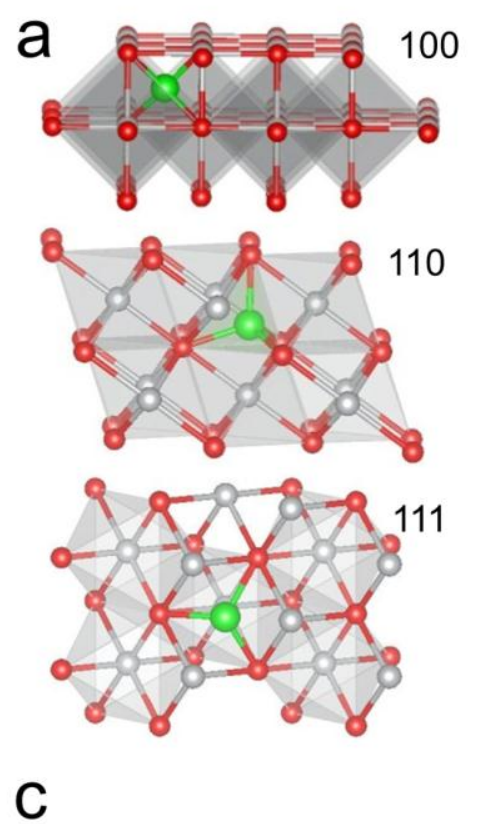

C

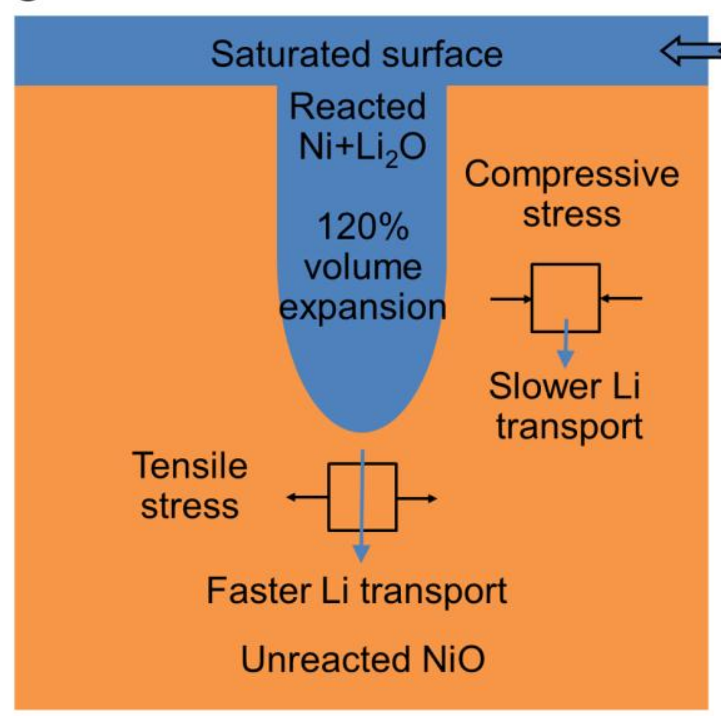

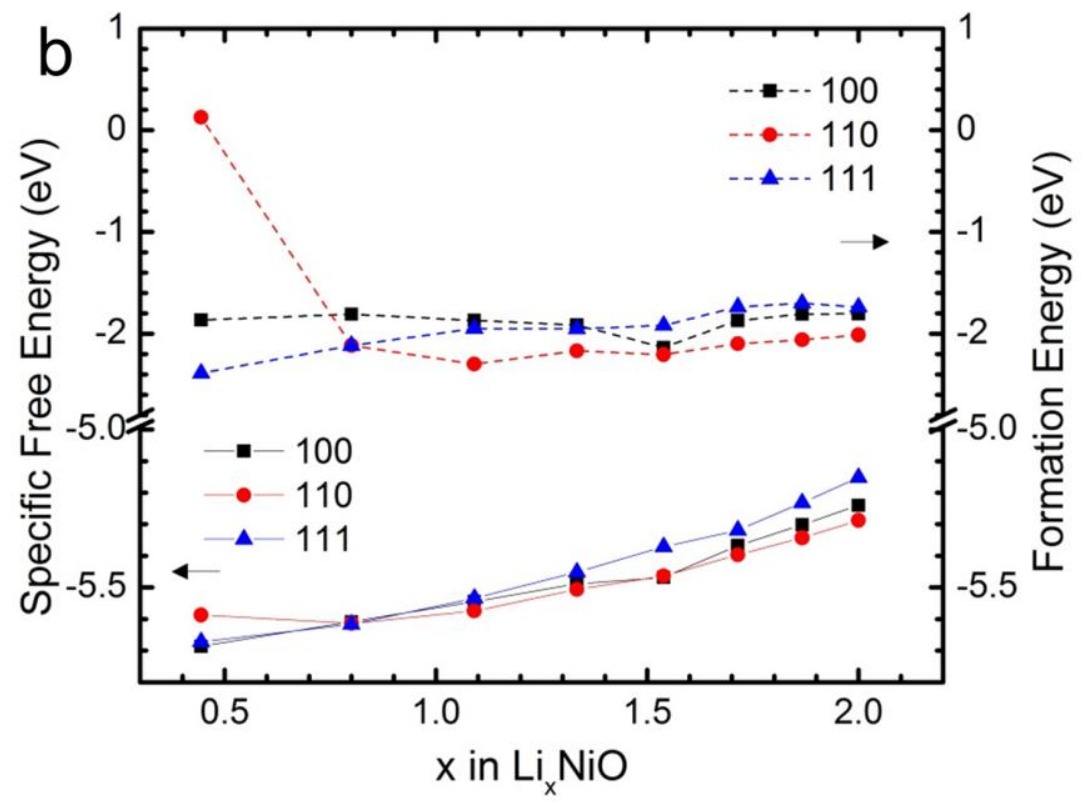

Surface

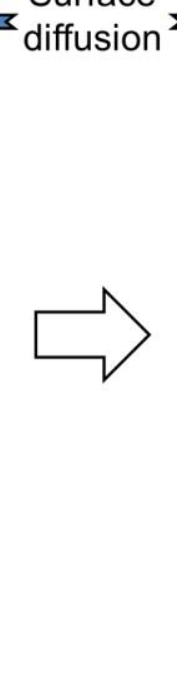

\section{Saturated surface}

Reacted

$\mathrm{Ni}+\mathrm{Li}_{2} \mathrm{O}$

$120 \%$

volume

Expansion

Figure 4. First-principles calculations and stress-induced growth. (a) The geometric feature of crystalline $\mathrm{NiO}$ for a lithium atom transport along the $\langle 100\rangle,\langle 110\rangle$, and $\langle 111\rangle$ directions (Nigray, O-red, Li-green). (b) Formation energy and specific free energy as a function of lithium content when $\mathrm{NiO}$ is lithiated along the $\langle 100\rangle,\langle 110\rangle$, and $\langle 111\rangle$ orientations. (c) Illustration of stress-induced self-sustaining finger growth. 